\title{
Characterization of active miniature inverted-repeat transposable elements in the peanut genome
}

\author{
Kenta Shirasawa $\cdot$ Hideki Hirakawa $\cdot$ Satoshi Tabata $\cdot$ Makoto Hasegawa \\ Hiroyuki Kiyoshima · Sigeru Suzuki · Sigemi Sasamoto - Akiko Watanabe · \\ Tsunakazu Fujishiro $\cdot$ Sachiko Isobe
}

Received: 12 September 2011/ Accepted: 5 January 2012/Published online: 1 February 2012

(C) The Author(s) 2012. This article is published with open access at Springerlink.com

\begin{abstract}
Miniature inverted-repeat transposable elements (MITEs), some of which are known as active nonautonomous DNA transposons, are found in the genomes of plants and animals. In peanut (Arachis hypogaea), AhMITE1 has been identified in a gene for fatty-acid desaturase, and possessed excision activity. However, the AhMITE1 distribution and frequency of excision have not been determined for the peanut genome. In order to characterize AhMITE1s, their genomic diversity and transposition ability was investigated. Southern blot analysis indicated high AhMITEl copy number in the genomes of $A$. hypogaea, A. magna and A. monticola, but not in A. duranensis. A total of 504 AhMITEls were identified from the MITE-enriched genomic libraries of A. hypogaea. The representative AhMITEls exhibited a mean length of $205.5 \mathrm{bp}$ and a GC content of $30.1 \%$, with AT-rich, 9 bp target site duplications and $25 \mathrm{bp}$ terminal inverted repeats. PCR analyses were performed using primer pairs designed against both flanking sequences of each AhMITE1. These analyses detected polymorphisms at 169 out of 411
\end{abstract}

Communicated by R. Varshney.

Electronic supplementary material The online version of this article (doi:10.1007/s00122-012-1798-6) contains supplementary material, which is available to authorized users.

K. Shirasawa $(\bowtie) \cdot$ H. Hirakawa $\cdot$ S. Tabata $\cdot$ S. Sasamoto

A. Watanabe $\cdot$ T. Fujishiro $\cdot$ S. Isobe

Department of Plant Genome Research, Kazusa DNA

Research Institute, 2-6-7 Kazusa-Kamatari, Kisarazu,

Chiba 292-0818, Japan

e-mail: shirasaw@kazusa.or.jp

M. Hasegawa $\cdot$ H. Kiyoshima $\cdot$ S. Suzuki

Peanut Plant Breeding Field, Chiba Prefectural Agriculture

and Forestry Research Center, 199 He, Yachimata,

Chiba 289-1113, Japan insertional loci in the four peanut lines. In subsequent analyses of 60 gamma-irradiated mutant lines, four $A h$ MITE1 excisions showed footprint mutations at the 109 loci tested. This study characterizes AhMITEls in peanut and discusses their use as DNA markers and mutagens for the genetics, genomics and breeding of peanut and its relatives.

\section{Introduction}

Transposable elements were first found in maize (McClintock 1951). They have been identified in both plants and animals (Feschotte et al. 2002). There are two types of transposable element, retrotransposons and DNA transposons. Retrotransposons move within genomes via RNA intermediates, using a 'copy-and-paste' mechanism. In contrast, the DNA of a DNA transposon is moved by a 'cut-and-paste' mechanism. The DNA transposons may be categorized into two groups, autonomous and non-autonomous elements. Autonomous elements contain regions encoding transposases which are necessary for the movement of transposons. Non-autonomous elements lack these coding sequences and must be activated by transposases supplied by autonomous elements. Most DNA transposons are flanked by terminal inverted repeats (TIRs), which function as receptor sites for the transposases (Feschotte et al. 2002). Target-site duplications (TSDs) are observed outside the TIRs.

Miniature inverted-repeat transposable elements (MITEs) are non-autonomous elements of less than $600 \mathrm{bp}$ in length. There are two major families of MITE, Tourist and Stowaway, as well as several minor families (Casa et al. 2000; Casacuberta et al. 1998; Charrier et al. 1999). Plant genomes usually contain between $10^{3}$ and $10^{5}$ copies 
(Feschotte et al. 2002). MITE mobility was demonstrated initially in rice (Jiang et al. 2003; Kikuchi et al. 2003; Nakazaki et al. 2003) and has been reported subsequently in other eukaryotes (Momose et al. 2010). It is likely that MITEs are one of the key factors accelerating eukaryotic evolution (Naito et al. 2009), since they tend to transpose into genes or their flanking regions (Feschotte et al. 2002), which may result in the disruption or promotion of gene expression.

Peanut (Arachis hypogaea) is an important food and oil crop. This allotetraploid species possesses an AABB genome derived from two diploids, most likely A. duranensis (AA) and A. ipaënsis (BB) (Kochert et al. 1996). On the basis of branching habit and branch length, peanuts are categorized into two subspecies: hypogaea and fastigiata; six varieties: hypogaea, hirsuta, fastigiata, vulgaris, aequatoriana, and peruviana; and four agronomic types: Virginia, Spanish, Valencia and Southeast-runner (Krapovickas and Gregory 1994, 2007). In contrast to its large phenotypic variation, the species exhibits extremely low genetic diversity, as revealed by polymorphism analyses using restriction fragment length polymorphism and simple sequence repeat (SSR) marker systems (Burow et al. 2001; Ferguson et al. 2004; He et al. 2003; Koilkonda et al. 2011; Moretzsohn et al. 2004, 2005, 2009; Proite et al. 2007).

Patel et al. (2004) reported that, following treatment with a chemical mutagen, MITE insertion caused functional disruption of the fatty-acid desaturase-encoding gene $a h F A D 2 B$. This MITE did not belong to the Tourist or Stowaway families but contained $9 \mathrm{bp}$ TSDs and $25 \mathrm{bp}$ TIRs, and was also present in multiple copies in the genome (Patel et al. 2004). These findings were similar to those of the Bigfoot family in Medicago (Charrier et al. 1999). AhMITE1, which exhibits sequence similarities with the MITE reported by Patel et al. (2004), has been excised subsequently from a single locus in spontaneous and induced mutants (Gowda et al. 2010, 2011). These reports suggest that AhMITEl transposes or is activated by ethyl methane sulfonate, gamma irradiation, adverse environmental conditions and tissue culture.

This investigation focused on the genomic diversity of peanut AhMITEl and its ability to transpose to provide a better understanding of the roles played by MITEs in the peanut genome and to develop tools for genetic and genomic studies. Following the collection of genomic fragments containing AhMITEls, nucleotide sequence analyses of AhMITE1 loci indicated that these elements clustered into six subfamilies. Insertional polymorphisms were detected by PCR analyses. The genomic distribution and transposition ability of AhMITEl elements were also investigated. The discussion includes the potential for using these elements as DNA markers and as mutagens for advanced molecular breeding programs such as markerassisted selection.

\section{Materials and methods}

Plant materials

Four peanut lines, including three Virginia types (A. hypogaea spp. hypogaea var. hypogaea cv. 'Nakateyutaka', 'YI-0311', and 'Satonoka') and one Spanish type ( $A$. hypogaea spp. fastigiata var. fastigiata cv. 'Kintoki'), were used for the construction of AhMITEl-enriched genomic libraries and screening of AhMITEl-insertion polymorphisms. The related species, A. duranensis (AA), A. magna (BB) and A. monticola (AABB), were also used in Southern blot analyses. For determining the transposition ability of AhMITE1, 'Nakateyutaka' seeds were treated with gamma irradiation $(10 \mathrm{~Gy} / \mathrm{h})$ for $20 \mathrm{~h}$ at the Institute of Radiation Breeding, National Institute of Agrobiological Sciences, Japan. In June 2009, $\mathbf{M}_{1}$ seeds were planted in a field at the Kazusa DNA Research Institute, Japan $\left(35^{\circ} 19^{\prime} 35^{\prime \prime} \mathrm{N}, 139^{\circ} 59^{\prime} 22^{\prime \prime} \mathrm{E}\right)$. For screening transposants, a single seed was collected from each $\mathrm{M}_{1}$ plant and these $\mathbf{M}_{2}$ seeds were planted into soil-containing pots, which were then cultivated in a plant-growth chamber. Genomic DNA was extracted from leaves using a DNeasy Plant Mini Kit (Qiagen).

\section{Southern blot analysis}

Digoxigenin-labeled AhMITE1 probes were prepared using a PCR DIG Labeling Mix (Roche Diagnostics, Switzerland). AhMITE1-containing DNA fragments from the $a h$ $F A D 2 B$ locus were cloned into pGEM $^{\circledR}$-T Easy (Promega) and used as templates for the PCR amplification of probes with the oligonucleotide primer (5'-AAGGTGGATACTACMATGAAGAT- $3^{\prime}$ ). Genomic DNA was digested with EcoRI and separated by electrophoresis in a $1.0 \%$ agarose gel. DNA fragments were transferred to a nylon membrane (Hybond $\mathrm{N}+$, GE Lifescience) and hybridized with digoxigenin-labeled probes at $65^{\circ} \mathrm{C}$ for $16 \mathrm{~h}$. Following hybridization, membranes were washed twice with $0.5 \times \mathrm{SSC}, 0.1 \% \mathrm{SDS}$ at $60^{\circ} \mathrm{C}$ for $20 \mathrm{~min}$. Signal detection was performed with a DIG Nucleic Acid Detection Kit (Roche Diagnostics, Switzerland).

Construction of AhMITE1-enriched genomic libraries and sequence analyses

Enrichment of genomic DNA fragments containing AhMITE1 transposons was performed as described by Nunome et al. (2006) with minor modifications. Biotinlabeled probes were prepared by PCR from the plasmid DNA used in the Southern blot analysis with the oligonucleotide primers (5'-AAGGTGGATACTACMATGAA GAT $-3^{\prime}$ ) labeled at the $5^{\prime}$ end with biotin. Genomic DNA 
was digested with nine restriction enzymes, i.e., $A f a \mathrm{I}, A l u \mathrm{I}$, HaeIII, HpyCH4V, MseI, PvuII, ScaI, SspI and XspI, to enhance the number of independent clones containing AhMITE1 sequences. Digested DNA fragments were ligated to linkers (5'-GTTTAGCCTTGTAGCAGAAGC- $3^{\prime}$ and $5^{\prime}$-GCTTCTGCTACAAGGCTAAACAAAA- $3^{\prime}$ phosphorylated at the $5^{\prime}$ end) using the LigaFast Rapid DNA Ligation System (Promega). Probes were then hybridized to the fragments and complementary sequences were collected using Dynal Magnetic Beads (Invitrogen). Using primers for the linker sequences, recovered DNA fragments were amplified by PCR and then ligated into pGEM$\mathrm{T}^{\circledR}$ Easy. Plasmids were introduced into Escherichia coli ElectroTen-blue (Stratagene) by electroporation. Following the amplification of DNA inserts with the Illustra TempliPhi DNA Amplification Kit (GE Lifescience), nucleotide sequences were determined using the BigDye Terminator Kit (Applied Biosystems) and an ABI 3730xl DNA sequencer (Applied Biosystems).

Computational processing and sequence analyses

Sequence data were subjected to base-calling with the PHRED program (Ewing et al. 1998; Ewing and Green 1998). Vector and linker sequences were masked with the CROSS_MATCH program using the parameters -minmatch 10 and -minscore 18 (Ewing and Green 1998). Masked and low quality bases generating Phred scores $<20$ were clipped using the TRIM2 program (-q $20-\times 10)$ (Huang et al. 2003) and sequences $>1 \mathrm{~kb}$ were excluded. The remaining sequences were compared with the CROSS_MATCH program (-minmatch 12 -penalty -2 -minscore 20) against AhMITEl sequences, and masked sequences were clipped using the TRIM2 program. Following trimming of the AhMITE1 sequences, the remaining flanking sequences were assembled with the CAP3 program using default parameters (Huang and Madan 1999). Sequences derived from the same loci were integrated into contigs, from which representative sequences were used for subsequent analyses. Similarity searches of AhMITE1 flanking sequences were performed against the NCBI $\mathrm{nr}$ (non-redundant amino acid sequences) database (http://www.ncbi.nlm.nih.gov) using the BLASTX program and an $E$ value cutoff of $\leq 1 e^{-4}$ (Altschul et al. 1997). The top hits are summarized in Table S1. For classification of the AhMITEls, a multiple sequence alignment of sequences from independent loci was performed using CLUSTALW with default parameters (Thompson et al. 1994). A dendrogram of the aligned sequences was constructed with the neighbor-joining algorithm using MEGA5 software (Tamura et al. 2011). For the analysis of polymorphic insertions, PRIMER3 software was used to design primer pairs based on AhMITEl flanking sequences to amplify 300-600 bp DNA fragments containing AhMITE1 loci (Rozen and Skaletsky 2000).

PCR amplification of AhMITE1 sites

PCR amplifications were performed using $0.5 \mathrm{ng}$ peanut genomic DNA in a $5 \mu$ reaction mix containing $1 \times$ PCR buffer (BIOLINE, UK), $3 \mathrm{mM} \mathrm{MgCl}_{2}, 0.04 \mathrm{U}$ BIOTAQ DNA polymerase (BIOLINE, UK), $0.2 \mathrm{mM}$ dNTPs and $0.8 \mu \mathrm{M}$ of each primer. The thermal cycling conditions were as follows: 1 min denaturation at $94^{\circ} \mathrm{C} ; 35$ cycles of $30 \mathrm{~s}$ denaturation at $94^{\circ} \mathrm{C}, 30 \mathrm{~s}$ annealing at $58^{\circ} \mathrm{C}$ and $1 \mathrm{~min}$ extension at $72^{\circ} \mathrm{C}$; and a final 3 min extension at $72^{\circ} \mathrm{C}$. PCR products were separated by electrophoresis in a $10 \%$ polyacrylamide gel with TBE buffer or with a micro-tip fragment analyzer (MultiNA, Shimadzu), according to the standard protocols. Gels were stained with ethidium bromide for the detection of DNA bands under UV illumination.

\section{Results}

Isolation and characterization of AhMITEls

by Southern blot analysis and sequencing analysis

To investigate the AhMITE1 family in the genomes of peanut and related species, Southern blot analyses were carried out using the digoxigenin-labeled AhMITEl fragments as probes. Multiple bands were detected in four lines of A. hypogaea, as well as in A. magna and A. monticola, while very faint bands were detected in A. duranensis (Fig. 1). The banding patterns indicated polymorphism between the four lines of A. hypogaea as well as between the four Arachis species, which suggests the presence of different AhMITEl insertion sites in each line.

AhMITE1-enriched genomic libraries were constructed using DNAs from three lines, i.e., 'Nakateyutaka', 'YI-0311', and 'Kintoki'. Nucleotide sequences were obtained from 8,736 clones and clustering analysis of the AhMITE1 flanking sequences indicated that 504 sequences were from independent AhMITE1 loci (Table S1, DDBJ accession numbers: DE998420-DE998923; also see http://marker.kazusa.or.jp).

To investigate the positions of AhMITE1 insertions in the peanut genome, the 504 flanking sequences were subjected to similarity searches against non-redundant amino acid sequences (NCBI nr: http://www.ncbi.nlm.nih.gov) using the BLASTX program. Out of the 504 sequences, 58 (11.5\%) showed significant sequence similarity to reported genes (Table S1).

To predict 9 bp TSDs as reported by Patel et al. (2004), comparison analyses were performed using sequences flanking the $5^{\prime}$ - and $3^{\prime}$ - ends of all 504 AhMITEl sequences. A total of 286 pairs of TSD sequences matched 


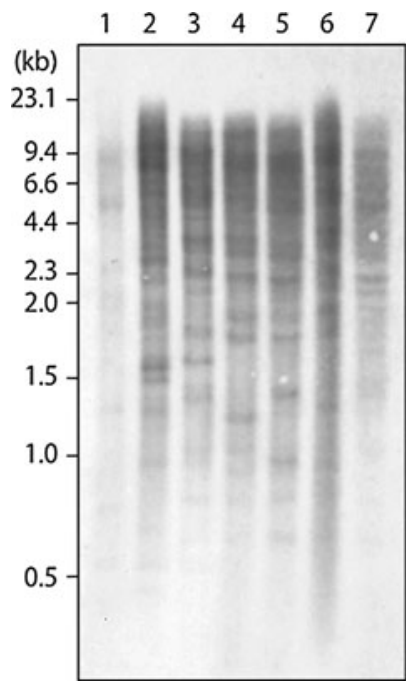

Fig. 1 Southern blot analysis of genomic DNA from Arachis species using AhMITE1 probes. 1, A. duranensis (AA genome); 2, A. magna (BB genome); 3, A. monticola (AABB genome); 4, A. hypogaea spp. hypogaea var. hypogaea cv. 'Nakateyutaka', Virginia type (AABB genome); 5, A. hypogaea spp. hypogaea var. hypogaea cv. 'YI-0311', Virginia type (AABB genome); 6, A. hypogaea spp. hypogaea var. hypogaea cv. 'Satonoka', Virginia type (AABB genome); 7, A. hypogaea spp. fastigiata var. fastigiata cv. 'Kintoki', Spanish type (AABB genome)

completely with non-conserved AT-rich sequences (93.4\%), while the remaining 218 pairs contained one to nine mismatches (Table S1). The 286 AhMITE1s containing completely matching TSDs were subjected to further analysis, since they presumably represented newer insertions, while the other 218 transposons contained mutations in the TSDs, TIRs and internal transposon sequences. The mean AhMITEl length was $205.5 \mathrm{bp}$ and sizes ranged from 201 to $223 \mathrm{bp}$, while the mean GC content was $30.1 \%$, which is lower than the Arachis hypogaea genome, i.e., 38.9\% (DDBJ accession number: FI498696-FI503143). No protein-encoding genes were predicted among the 286 sequences, which suggests that these AhMITE1s are nonautonomous. Cluster analysis of the AhMITE1 sequences (201-223 bp in length) grouped the elements into six families (AhMITE1-1 to AhMITE1-6; Fig. 2).
The ends of all 286 pairs of AhMITEl sequences were compared to identify TIRs, which were found to be $25 \mathrm{bp}$ in length and matched the sequences reported by Patel et al. (2004). The 572 TIR sequences were classified into seven consensuses (TIR1-TIR7) and 14 unique sequences (Fig. 3a). Five combinations of seven consensus sequences were found in 273 AhMITE 1s, with pairs of TIR1s identified in 29 sequences in addition to the original AhMITEl (Patel et al. 2004; Fig. 3b, Table S1). A combination of TIR2 and TIR3 was found most commonly, i.e., in 142 AhMITE1-2s, followed by TIR4-TIR5 in 55 AhMITE1-3s, TIR6-TIR7 in 24 AhMITE1-4s, TIR1-TIR6 in 13 AhMITE1-5s, and TIR6-TIR7 in 10 AhMITE1-6s (Fig. 3b; Table S1). The remaining 13 AhMITEl sequences exhibited unique combinations of TIRs that differed from those described above. Among these different groups, sequence diversity was greatest within the AhMITE1-2 sequences (Fig. 4).

AhMITE1 insertion polymorphisms among cultivated peanut lines

To investigate AhMITE1 insertion polymorphisms among peanut lines, 504 primer pairs were designed against both flanking sequences of each AhMITE1 (Table S1). Among the primer pairs tested, 240 and 171 generated single and double DNA bands, respectively. The double bands likely derived from homoeologous regions in the $\mathrm{A}$ and $\mathrm{B}$ genomes. Of the 411 primer pairs that produced amplicons, 169 showed polymorphism between the four lines, i.e., 'Nakateyutaka', 'YI-0311', 'Satonoka' and 'Kintoki' (Fig. 5; Table 1, Table S1). The mean number of polymorphic sites between any two lines was 90.3 (22.0\%), while means of $55.0(13.4 \%)$ and $125.6(30.6 \%)$ were exhibited among Virginia lines and between Virginia and Spanish types, respectively (Table 1).

Transposition ability of AhMITE1s in peanut

Since MITEs have been shown to activate under stress conditions (Kikuchi et al. 2003; Lin et al. 2006; Nakazaki et al. 2003; Shan et al. 2005), AhMITEl transposition

\begin{abstract}
AhMITE 1-1 AhMITE 1-2 AhMITE 1-3 AhMITE 1-4 AhMITE 1-5 AhMITE 1-6

AhMITE 1-1 AhMITE 1-2 AhMITE 1-3 AhMITE 1-4 AhMITE $1-5$ AhMITE 1-6

GGGAGCTACTCAAATGAAGATGCAAAAAACATCTTTTTATGAAGATGCTTTGTATAAAAGTGTGATTTATTGATTT--GGCCACACTTCAAATAAAAACAACAC GGTGGCTACTCCAATGAAGATTTAATGATTATCATCATGTGAAGATACATCATTTTGACCATTGGATGATAGATTGTAGGGCTTGATTTGATTTGAAGTAAAAG GGTGGATACTACAATGAAGATGGCATAATTGTCTTCATATGAGTATATTTCTTTTTGACCTTTGGATGATGGATTGTATGGTTAGATTTTGATATTTATAAAAG GGCAACTACTCAAATGAAGATGCTAAAAACATCTTTTTATGAAGATTCTTGTGTTAAAAGTGTACTTTGTTTGTTT--AGCCACACTTTAAA-AAAAATAACAC GGTGGATACTACAATGAAGATGGCAAAAATATCTTCTCATGAAGATGCTTTGGTTAAAAGTGTGGTTTATTTATTT--AGCCACACTTTAAACAAAAATAACAC GGCAACTACTCAAATGAAGATGGCAAAAACATCTTTTTATGAAGATGCTTTGTTTAAAAGTGTGATTTATTTATTT--AGCCACACTTTAAACAAAGACAACAC

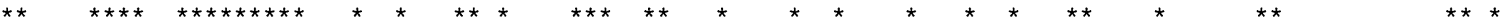

TTTTATAACATATCAAAATCTAACCCTACACTCCATCATCTAAGGGTCAAAAAGAAAAATCATCACATGAAGACAATTATAATATCTTCATGGGAGTACCCACC TGTTACTTTTATTTAAAATGTTGCTAAATAA---ATAAGTTACACTTTTTGAACAAGGATCATCATGAGAAGATGTTTTTGCCATCTTCATGGGAGTAGTTGCC TGTTGTTTTTGTTTAAAGTGTGGCTAAATAA---ATAAACCACACTTTTAACCAAAGCATCTTCATGAGAAGATATTTTTGCCATCTTCATTGTAGTATCCACC TTTTGTAACACATCAAAATCAAACCATACATTTCATCATCTAATGGTAAAAAAGAAGCATCTTCATATGAAGACAATAATAAAATCTTCATGGTAGTATCCACC TTTTATAA-ATATCAAAATCTAACCATACAATCCATCATCCAAGGGTCAAAAAGAAATATATTCACATGAAGACAATTATAAAATCTTCATTGTAGTATCCACC TTTTATAA-ATATCAAAATCTAACCATACAATCCATCATCCAAAGGTCAAAAAAAAATATTTTCACATGAAGACAATTATAAAATCTTCATTGTAGTATCCACC

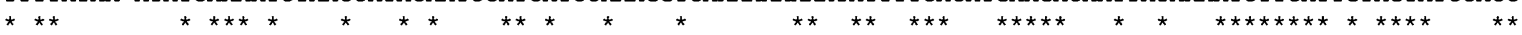

Fig. 2 Consensus sequences of AhMITE1 subfamilies. Asterisks indicate the positions of conserved bases among the six subfamilies 
a

TIR1

TIR2

TIR3

TIR4

TIR5

TIR6

TIR7

b

AhMITE 1-1 :

TIR1 5'-GGTGGATACTACAATGAAGATGGCA |||||||||||||||||||||||||

TIR1

5 '-GgTgGatactacAatgaAgATGGCA-3' 5 '-GGGAGCTACTCAAATGAAGATGCAA-3' 5 ' -GGTGGGTACTCCCATGAAGATATTA-3 ' 5 '-GGTGGCTACTCCAATGAAGATTTAA-3' 5 '-GGCAACTACTCCCATGAaGATGSCA-3' 5 '-GgTgGatACTACMATGAAGATTTTA-3' 5 '-GGCAACTACTCAAATGAAGATGSYA-3'

\section{AHMITE 1-2 :}

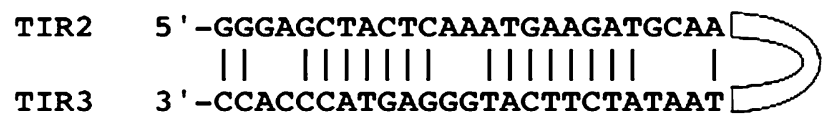

AhMITE 1-3 :

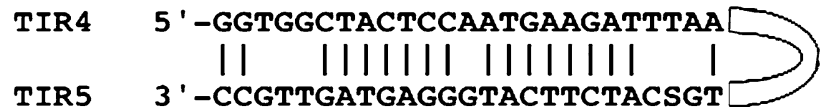

AhMITE1-4 and AhMITE1-6:

TIR6

5 ' -GGTGGATACTACMATGAAGATTTTA | | ||| :||||||| :

TIR7 3 '-CCGTTGATGAGTTTACTTCTACSRT

AhMITE 1-5:

TIR1 5'-GgTGgATACTACAATGAAGATGGCA

TIR6

Fig. 3 Terminal-inverted repeat sequences of AhMITE1s. a Seven TIR consensus sequences. $\mathrm{S}, \mathrm{M}$ and $\mathrm{Y}$ show $\mathrm{G} / \mathrm{C}, \mathrm{A} / \mathrm{C}$ and $\mathrm{C} / \mathrm{T}$, respectively. b Five TIR pairing patterns. Complementary matched and semi-complementary matched bases are delimited by lines and colons, respectively. Arms on the right side represent AhMITE1 internal sequences. $\mathrm{S}, \mathrm{M}, \mathrm{R}$ and $\mathrm{K}$ show $\mathrm{G} / \mathrm{C}, \mathrm{A} / \mathrm{C}, \mathrm{A} / \mathrm{G}$ and $\mathrm{G} / \mathrm{T}$, respectively

activity was investigated in peanut mutants generated by gamma irradiation. 'Nakateyutaka' seeds were gammairradiated (200 Gy) and then planted out as an $\mathrm{M}_{1}$ population. Of the $206 \mathrm{M}_{1}$ seeds, 125 died before or just after germination, while 14 grew but were sterile. The remaining $67 \mathrm{M}_{1}$ plants, i.e., G01-G67, grew normally and produced self-pollinated seeds. A single seed was collected from each $\mathrm{M}_{1}$ plant and these seeds were sown as the $\mathrm{M}_{2}$ plant generation. Leaves from $60 \mathrm{M}_{2}$ seedlings were collected for DNA analysis. Leaves from seven seedlings (G25, G33, G36, G37, G39, G40, and G41) were omitted from the analysis because the $\mathrm{M}_{2}$ seeds failed to germinate.

In the $60 \mathrm{M}_{2}$ plants, AhMITE1-containing genomic regions were PCR amplified using 109 primer pairs, all of which amplified 'Nakateyutaka' alleles with AhMITE1 insertions (Table S1). Four of the primer pairs amplified

lower molecular weight fragments in a part of the $\mathrm{M}_{2}$ plants. Among the $\mathrm{M}_{2}$ plants, a homozygous AhMITE1absent allele (G51) was identified at the AhTE0433 locus, a heterozygous mutant allele (G13) was observed at the AhTE0426 locus and two heterozygous mutant alleles (G13 and G49) were found at the AhTE0121 locus. An extraordinarily high frequency of $\mathrm{M}_{2}$ plants (14 of 60; G04, G06, G10, G18, G21, G23, G27, G32, G34, G45, G53, G58, G62, and G67) exhibited homozygous mutant alleles at the AhTE0047 locus. It was confirmed subsequently that some of these lines contained mutant alleles at the AhTE0047 locus prior to gamma irradiation, which indicates the presence of polymorphism within normal 'Nakateyutaka' lines.

To examine the mutation events in the four $\mathrm{M}_{2}$ mutant loci, nucleotide sequence comparisons were performed between targeted regions in the 18 mutated $\mathrm{M}_{2}$ plants and the four peanuts lines, i.e., 'Nakateyutaka', 'Kintoki', 'Satonoka' and 'YI-0311'. At the AhTE0433 locus, AhMITE1 deletion and a single-base T/C substitution were found in the $\mathrm{M}_{2}$ mutant (Fig. 6a), while the AhTE0426 and AhTE0047 loci exhibited two di-nucleotide insertions and AhMITE1 excisions (Fig. 6b, c). At the AhTE0426 locus, the AA insertion might have derived from TSD, while the GT insertion may have arisen from the end of AhMITEl and TSD at the AhTE0047 locus. GT insertions were observed in 'Nakateyutaka' lines that were not treated with gamma irradiation. At the AhTE0121 locus, a T/C mutation was identified between 'Kintoki' and all the other lines, while an AhMITE1 deletion was found at the flanking sequence of the TSD in the mutant $\mathrm{M}_{2}$ line (Fig. 6d). This mutation may have been induced at the time of AhMITEl insertion. All of the investigated alleles showed AhMITE 1 excisions and base substitutions or insertions that represent probable footprint mutations.

\section{Discussion}

In the present study, 504 AhMITEls and their flanking sequences were isolated from the peanut genome and characterized. The AhMITE1s exhibited a mean length of $205.5 \mathrm{bp}$ and a GC content of $30.1 \%$, findings similar to those reported previously for peanut MITEs (Patel et al. 2004). Complete conservation of TIRs was observed in AhMITE1-1, but not in AhMITE1-2 to -6. The AT-rich, 9 bp TSDs were different from Tourist and Stowaway but similar to the Bigfoot family in Medicago (Charrier et al. 1999), as described by Patel et al. (2004).

Southern blot analysis revealed multiple AhMITE1 copies in the genomes of A. magna (BB), A. monticola (AABB) and A. hypogaea (AABB), but not in the genome of $A$. duranensis (AA) (Fig. 1). This result suggests that 


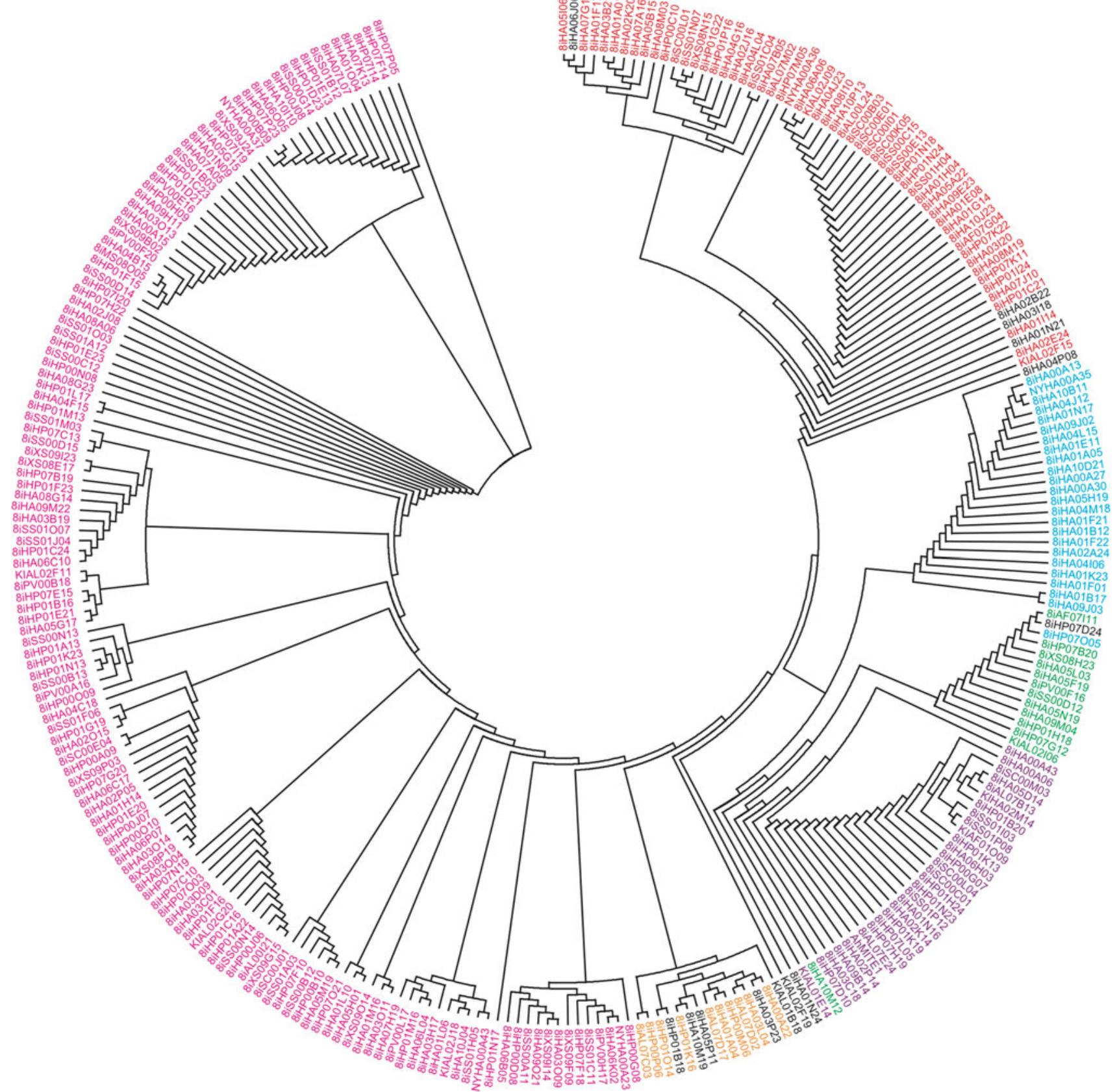

Fig. 4 AhMITE1 phylogenetic tree based on genetic distances calculated with the neighbor-joining method. Subfamilies of AhMITE1-1, -2, -3, -4, -5, and -6 are shown in purple, pink, red, blue,

AhMITEl elements have amplified in diploid species of the $\mathrm{B}$ genome and then transferred to tetraploid species. The tetraploid A. hypogaea was generated by hybridization between two A and B diploids. Since differences in $A h$ MITE1 insertion positions were observed not only among the four Arachis species but also between the four A. hypogaea lines, it is likely that AhMITE1 transposition occurred both before and after the generation of $A$. hypogaea. green and orange letters, respectively. AhMITE1s in black letters were not classified into any subfamilies. AhMITE1 is a sequence reported by Patel et al. (2004) (color figure online)

The AhMITE1 insertion frequency into gene-containing regions was $11.5 \%$. However, since BLASTX analysis does not detect AhMITE1 transpositions into promoter, intron or untranscribed regions, a higher than $11.5 \%$ frequency of insertions into gene regions might be expected, which is a higher frequency than by random transposition. Due to genome size, the gene regions in the peanut genome $(2.8 \mathrm{~Gb}$ ) should be a much lower ratio than $11.5 \%$ (Yüksel and Paterson 2005) since the Lotus japonicas (472 Mb 
Fig. 5 Polyacrylamide gel analysis of insertional polymorphisms in four cultivated peanut lines. Six markers were used. $S$ 'Satonoka', $K$ 'Kintoki'
$N$ 'Nakateyutaka', $Y$ 'YI-0311',

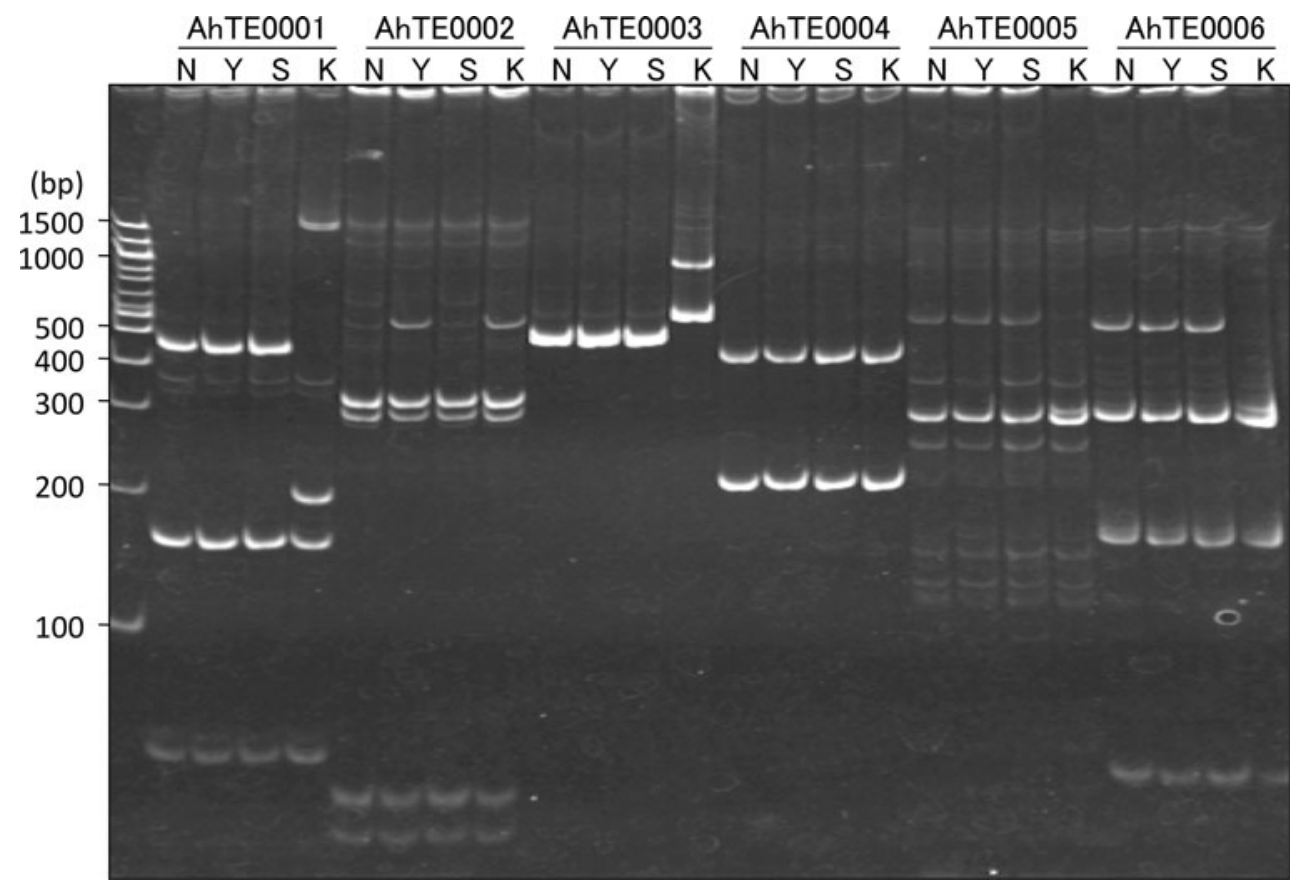

Table 1 Number and percentage of polymorphic markers among four peanut lines

\begin{tabular}{llr}
\hline Combinations & $\begin{array}{l}\text { No. of polymorphic } \\
\text { markers }^{\mathrm{a}}\end{array}$ & $\%$ \\
\hline Av. among four lines & 90.3 & 22.0 \\
Av. among Virginia types & 55.0 & 13.4 \\
Nakateyutaka vs. YI-0311 & 58 & 14.1 \\
Nakateyutaka vs. Satonoka & 30 & 7.3 \\
YI-0311 vs. Satonoka & 77 & 18.7 \\
Av. between Virginia and Spanish types & 125.6 & 30.6 \\
Nakateyutaka vs. Kintoki & 124 & 30.2 \\
YI-0311 vs. Kintoki & 147 & 35.8 \\
Satonoka vs. Kintoki & 106 & 25.8 \\
\hline
\end{tabular}

a The total number of primer pairs tested was 411

genome) ratio is calculated to be $9.4 \%$ (Sato et al. 2008). In maize and rice, MITEs have been reported to insert preferentially into genic regions (Bureau and Wessler 1994; Jiang et al. 2003), and similar findings have been revealed by whole genome sequencing studies in rice, sorghum and L. japonicus (International Rice Genome Sequencing Project 2005; Paterson et al. 2009; Sato et al. 2008). Gene function or expression may be disrupted or altered by transposition events, as well as by other genetic modifications such as genome rearrangement and duplication/ deletions, and point mutations. These modifications might contribute to the divergence of many plant species, including those in the genus Arachis.

It is clear that at least three AhMITE1s, i.e., those at the AhTE0433, AhTE0426 and AhTE0121 loci, were activated following gamma irradiation. Footprint mutations were detected at all of the empty sites investigated (Fig. 6). These findings imply that AhMITE1 transposition may be activated spontaneously or by gamma irradiation. The frequency of the de novo excision could be calculated to be 0.00023 [ $=3$ excision events/ $(60$ lines $\times 2$ haploids $\times 109$ loci)]. This value is similar to that of rice mPing under the normal condition (Monden et al. 2009). On the other hand, 14 lines were homozygous for empty alleles at the AhTE0047 locus, and the remaining 46 lines were homozygous for insert-containing alleles. These high frequencies of homozygous mutations suggests that AhMITE1 excision occurred in a single or a small number of 'Nakateyutaka' plants during the breeding process, or when the population size was relatively small, i.e., just after this line was released in 1980 from the Chiba Prefectural Agriculture and Forestry Research Center, Japan. The later distribution of a mixed stock would explain the presence of the mutated allele within some of the 'Nakateyutaka' line. Although it is not possible to estimate spontaneous excision frequency at the AhTE0047 locus using the present data, this finding may indicate that AhMITE1 remains slightly active in normal plants.

In polyploid species, reverse genetics is an effective strategy for functional genomics, as well as for mutationbased breeding. This is because single gene mutations do not necessarily confer phenotypic variation due to functional complementation by homoeologous genes. A combination or pyramiding of homoeologous mutated genes would be expected to result in phenotypic changes. In plants and animals, the target-induced local lesions in genome (TILLING) technique is a widely-used reverse 


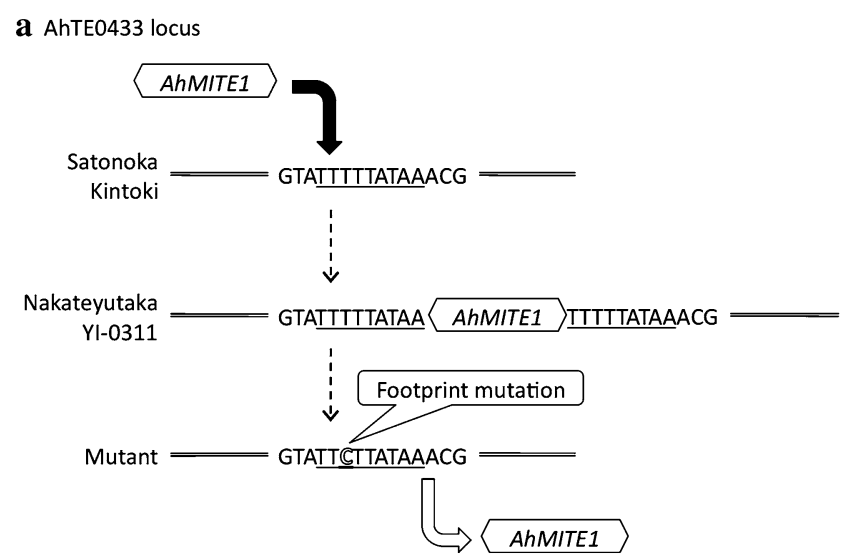

c AhTE0047 locus

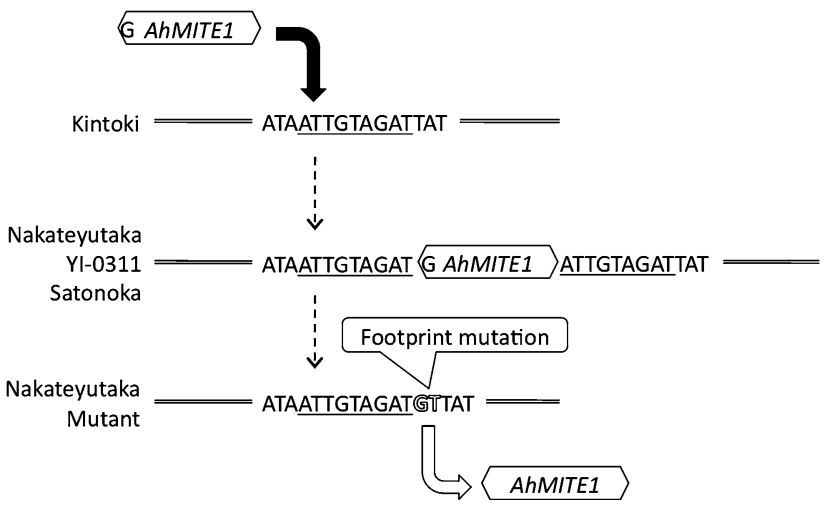

Fig. 6 Sequences of target site duplications and flanking regions at the AhTE0433 (a), AhTE0426 (b), AhTE0047 (c) and AhTE0121 (d) loci. The nucleotides involved in target site duplications and

genetic tool (Henikoff et al. 2004) that has been employed for allergen reduction and the improvement of quality traits in peanut (Knoll et al. 2011). In addition to TILLING, transposons and retrotransposons are also useful mutagens for reverse genetic approaches in functional genomics and mutation breedings (Gierl and Saedler 1992). In particular, MITEs tend to transpose into gene and promoter regions, which is a desirable characteristic for mutagens. The retrotransposon FIDEL is well-characterized in peanut (Nielen et al. 2010) and, like AhMITEls, FIDELs may be valuable tools for functional genomics. However, at present, there is no evidence to suggest that FIDEL is active.

Although there has been considerable effort for the development of DNA markers for peanut, e.g., genomic and expressed sequence tag SSRs, the efficiency of polymorphic marker production is very low due to the narrow genetic diversity (Ferguson et al. 2004; He et al. 2003; Koilkonda et al. 2011; Moretzsohn et al. 2004, 2005, 2009; Proite et al. 2007). In both plants and animals, different patterns of MITE insertion in germplasms or individuals have also been used as DNA markers (Bonin et al. 2008; Monden et al. 2009; Grzebelus et al. 2009). Therefore, it is worth considering the use of AhMITEls as DNA markers. b AhTE0426 locus

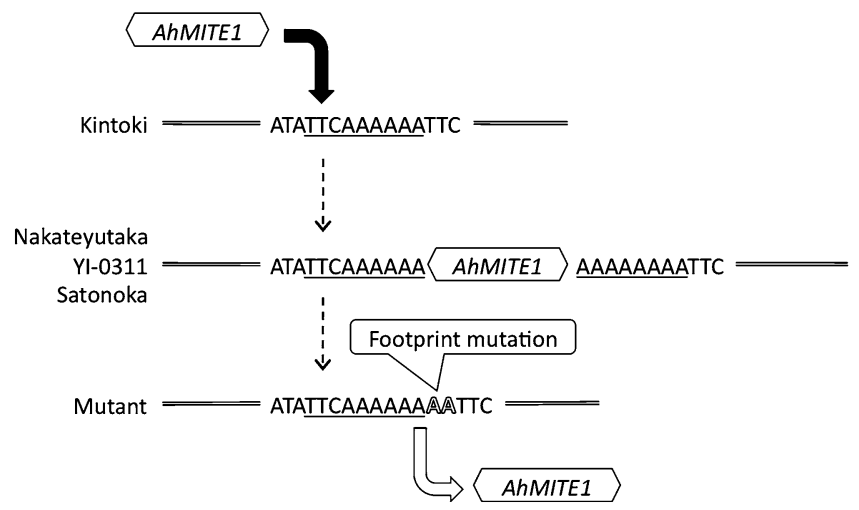

d AhTE0121 locus

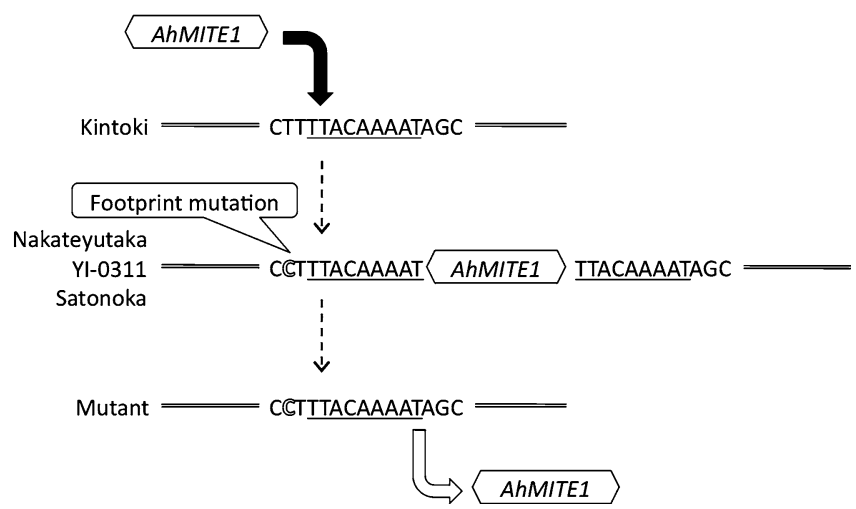

footprint mutations are underlined and outlined, respectively. Black and white arrows show AhMITE1 insertion and excision events, respectively

In this study, amplified DNA fragments exhibited an approximately $200 \mathrm{bp}$ difference in size, which corresponds to the size of the AhMITEls (Fig. 5). This finding suggests that these polymorphisms derive from the presence or absence of AhMITEl at the loci, and the sequencing analysis of four loci has confirmed this prediction (Fig. 6). For the construction of linkage maps, evolutionary studies on the Arachis genome, and as a convenient tool for molecular breeding, the stable inheritance and the genome-wide distribution of AhMITE1 loci are required for their use as DNA markers, while the frequency of transposition is unlikely to be a concern for linkage analysis (Monden et al. 2009).

In conclusion, this study has examined the characteristics of AhMITE1s in the peanut genome, as well as investigating the flanking genomic sequence, insertional polymorphisms in cultivars and transposition ability. These findings will contribute to our understanding of peanut diversity and assist in the progression of genetics, genomics and the breeding of peanut and its relatives.

Acknowledgments This work was supported by the foundation of the Kazusa DNA Research Institute. 
Open Access This article is distributed under the terms of the Creative Commons Attribution License which permits any use, distribution, and reproduction in any medium, provided the original author(s) and the source are credited.

\section{References}

Altschul SF, Madden TL, Schaffer AA, Zhang J, Zhang Z, Miller W, Lipman DJ (1997) Gapped BLAST and PSI-BLAST: a new generation of protein database search programs. Nucleic Acids Res 25:3389-3402

Bonin A, Paris M, Despres L, Tetreau G, David JP, Kilian A (2008) A MITE-based genotyping method to reveal hundreds of DNA polymorphisms in an animal genome after a few generations of artificial selection. BMC Genomics 9:459

Bureau TE, Wessler SR (1994) Mobile inverted-repeat elements of the Tourist family are associated with the genes of many cereal grasses. Proc Natl Acad Sci USA 91:1411-1415

Burow MD, Simpson CE, Starr JL, Paterson AH (2001) Transmission genetics of chromatin from a synthetic amphidiploid to cultivated peanut (Arachis hypogaea L.): broadening the gene pool of a monophyletic polyploid species. Genetics 159:823-837

Casa AM, Brouwer C, Nagel A, Wang L, Zhang Q, Kresovich S, Wessler SR (2000) The MITE family Heartbreaker (Hbr): molecular markers in maize. Proc Natl Acad Sci USA 97:10083-10089

Casacuberta E, Casacuberta JM, Puigdomenech P, Monfort A (1998) Presence of miniature inverted-repeat transposable elements (MITEs) in the genome of Arabidopsis thaliana: characterisation of the Emigrant family of elements. Plant J 16:79-85

Charrier B, Foucher F, Kondorosi E, d'Aubenton-Carafa Y, Thermes C, Kondorosi A, Ratet P (1999) Bigfoot: a new family of MITE elements characterized from the Medicago genus. Plant $\mathrm{J}$ 18:431-441

Ewing B, Green P (1998) Base-calling of automated sequencer traces using phred. II. Error probabilities. Genome Res 8:186-194

Ewing B, Hillier L, Wendl MC, Green P (1998) Base-calling of automated sequencer traces using phred. I. Accuracy assessment. Genome Res 8:175-185

Ferguson ME, Burow MD, Schulze SR, Bramel PJ, Paterson AH, Kresovich S, Mitchell S (2004) Microsatellite identification and characterization in peanut (A. hypogaea L.). Theor Appl Genet 108:1064-1070

Feschotte C, Jiang N, Wessler SR (2002) Plant transposable elements: where genetics meets genomics. Nat Rev Genet 3:329-341

Gierl A, Saedler H (1992) Plant-transposable elements and gene tagging. Plant Mol Biol 19:39-49

Gowda MVC, Bhat RS, Motagi BN, Sujay V, Kumari V, Sujatha B (2010) Association of high-frequency origin of late leaf spot resistant mutants with AhMITE1 transposition in peanut. Plant Breed 129:567-569

Gowda MVC, Bhat RS, Sujay V, Kusuma P, Varshakumari Bhat S, Varshney RK (2011) Characterization of AhMITE1 transposition and its association with the mutational and evolutionary origin of botanical types in peanut (Arachis spp.). Plant Syst Evol 291:153-159

Grzebelus D, Gladysz M, Macko-Podgorni A, Gambin T, Golis B, Rakoczy R, Gambin A (2009) Population dynamics of miniature inverted-repeat transposable elements (MITEs) in Medicago truncatula. Gene 448:214-220

He G, Meng R, Newman M, Gao G, Pittman RN, Prakash CS (2003) Microsatellites as DNA markers in cultivated peanut (Arachis hypogaea L.). BMC Plant Biol 3:3
Henikoff S, Till BJ, Comai L (2004) TILLING. Traditional mutagenesis meets functional genomics. Plant Physiol 135:630-636

Huang X, Madan A (1999) CAP3: a DNA sequence assembly program. Genome Res 9:868-877

Huang X, Wang J, Aluru S, Yang SP, Hillier L (2003) PCAP: a whole-genome assembly program. Genome Res 13:2164-2170

International Rice Genome Sequencing Project (2005) The map-based sequence of the rice genome. Nature 436:793-800

Jiang N, Bao Z, Zhang X, Hirochika H, Eddy SR, McCouch SR, Wessler SR (2003) An active DNA transposon family in rice. Nature 421:163-167

Kikuchi K, Terauchi K, Wada M, Hirano HY (2003) The plant MITE $m$ Ping is mobilized in anther culture. Nature 421:167-170

Knoll JE, Ramos ML, Zeng Y, Holbrook CC, Chow M et al (2011) TILLING for allergen reduction and improvement of quality traits in peanut (Arachis hypogaea L.). BMC Plant Biol 11:81

Kochert G, Stalker HT, Gimenes M, Galagro L, Lopes CR, Moore K (1996) RFLP and cytogenetic evidence on the origin and evolution of allotetraploid domesticated peanut, Arachis hypogaea (Leguminosae). Am J Bot 83:1282-1291

Koilkonda P, Sato S, Tabata S, Shirasawa K, Hirakawa H et al (2011) Large-scale development of expressed sequence tag-derived simple sequence repeat markers and diversity analysis in Arachis spp. Mol Breed. doi:10.1007/s11032-011-9604-8

Krapovickas A, Gregory W (1994) Taxonomia del genero Arachis (Leguminosae). Bonplandia 8:1-186

Krapovickas A, Gregory W (2007) Taxonomy of the genus Arachis (Leguminosae). Bonplandia 16:1-205

Lin X, Long L, Shan X, Zhang S, Shen S, Liu B (2006) In planta mobilization of mPing and its putative autonomous element Pong in rice by hydrostatic pressurization. J Exp Bot 57:2313-2323

McClintock B (1951) Chromosome organization and genic expression. Cold Spring Harb Symp Quant Biol 16:13-47

Momose M, Abe Y, Ozeki Y (2010) Miniature inverted-repeat transposable elements of Stowaway are active in potato. Genetics 186:59-66

Monden Y, Naito K, Okumoto Y, Saito H, Oki N et al (2009) High potential of a transposon mPing as a marker system in japonica $\times$ japonica cross in rice. DNA Res 16:131-140

Moretzsohn MC, Hopkins MS, Mitchell SE, Kresovich S, Valls JF, Ferreira ME (2004) Genetic diversity of peanut (Arachis hypogaea L.) and its wild relatives based on the analysis of hypervariable regions of the genome. BMC Plant Biol 4:11

Moretzsohn MC, Leoi L, Proite K, Guimaraes PM, Leal-Bertioli SC et al (2005) A microsatellite-based, gene-rich linkage map for the AA genome of Arachis (Fabaceae). Theor Appl Genet 111:1060-1071

Moretzsohn MC, Barbosa AV, Alves-Freitas DM, Teixeira C, LealBertioli SC et al (2009) A linkage map for the B-genome of Arachis (Fabaceae) and its synteny to the A-genome. BMC Plant Biol 9:40

Naito K, Zhang F, Tsukiyama T, Saito H, Hancock CN et al (2009) Unexpected consequences of a sudden and massive transposon amplification on rice gene expression. Nature 461:1130-1134

Nakazaki T, Okumoto Y, Horibata A, Yamahira S, Teraishi M et al (2003) Mobilization of a transposon in the rice genome. Nature 421:170-172

Nielen S, Campos-Fonseca F, Leal-Bertioli S, Guimaraes P, Seijo G et al (2010) FIDEL - a retrovirus-like retrotransposon and its distinct evolutionary histories in the A- and B-genome components of cultivated peanut. Chromosom Res 18:227-246

Nunome T, Negoro S, Miyatake K, Yamaguchi H, Fukuoka H (2006) A protocol for the construction of microsatellite enriched genomic library. Plant Mol Bio Rep 24:305-312 
Patel M, Jung S, Moore K, Powell G, Ainsworth C, Abbott A (2004) High-oleate peanut mutants result from a MITE insertion into the FAD2 gene. Theor Appl Genet 108:1492-1502

Paterson AH, Bowers JE, Bruggmann R, Dubchak I, Grimwood J et al (2009) The Sorghum bicolor genome and the diversification of grasses. Nature 457:551-556

Proite K, Leal-Bertioli SC, Bertioli DJ, Moretzsohn MC, da Silva FR, Martins NF, Guimaraes PM (2007) ESTs from a wild Arachis species for gene discovery and marker development. BMC Plant Biol 7:7

Rozen S, Skaletsky H (2000) Primer3 on the WWW for general users and for biologist programmers. Methods Mol Biol 132:365-386

Sato S, Nakamura Y, Kaneko T, Asamizu E, Kato T et al (2008) Genome structure of the legume, Lotus japonicus. DNA Res $15: 227-239$
Shan X, Liu Z, Dong Z, Wang Y, Chen Y et al (2005) Mobilization of the active MITE transposons mPing and Pong in rice by introgression from wild rice (Zizania latifolia Griseb.). Mol Biol Evol 22:976-990

Tamura K, Peterson D, Peterson N, Stecher G, Nei M, Kumar S (2011) MEGA5: molecular evolutionary genetics analysis using maximum likelihood, evolutionary distance, and maximum parsimony methods. Mol Biol Evol 28:2731-2739

Thompson JD, Higgins DG, Gibson TJ (1994) CLUSTAL W: improving the sensitivity of progressive multiple sequence alignment through sequence weighting, position-specific gap penalties and weight matrix choice. Nucleic Acids Res 22:4673-4680

Yüksel B, Paterson AH (2005) Construction and characterization of a peanut HindIII BAC library. Theor Appl Genet 111:630-639 